\title{
Numerical simulation of plane flow field and the force on the inner rod induced by planetary motion of the rod string
}

\author{
Zhang Yang ${ }^{1,2}$, Dong Shimin ${ }^{1,2, *}$, Li Qin $^{1}$, Wang Zhe ${ }^{1}$, Yang Yu ${ }^{1}$, Wu Ruiqing ${ }^{1}$, Li Bingyi $^{1}$ \\ ${ }^{1}$ School of Mechanical Engineering, Yanshan University, Qinhuangdao 066004, China \\ ${ }^{2}$ Hebei Province Lightweight Structure Design \& Manufacturing Technology Innovation Center, Qinhuangdao 066004, China
}

\begin{abstract}
In order to a the flow of the plane flow field induced by the inner rod rotates and revolves in the cylinder, the Fluent software is used to numerically simulate the plane flow field of the eccentric annulus generated by the planetary motion of the rod string and based on the superposition principle. The velocity distribution and secondary flow of the two flow fields, as well as the fluid force on the inner rod are analyzed. The calculation results show that the flow field induced by the eccentric rotation of the inner rod and the selfrotation of the outer cylinder is quite different from the planetary motion of the inner rod. When rotation of the inner rod has the same direction with the revolution direction, the fluid velocity distribution near the wall of the inner rod is that the velocity on the narrow space side of the annulus is large, and on the wide space side is small. There is a critical value of eccentricity for secondary flow appears when the eccentricity is greater than this value. When rotation of the inner rod is contrary to the revolution, the fluid velocity distribution near the wall of the inner rod is that the velocity on the wide space side of the annulus is large, on the narrow space side is small. Different eccentricity has obvious secondary flow phenomenon where appears in a wide gap and close to the inner rod. When the inner rod revolves, there is a critical value of eccentricity, the inner rod is pushed outward by the fluid force when the eccentricity is less than this critical value. On the contrary, the inner rod is pushed inward. When rotation and revolution are reversed, the critical value of eccentricity increases, when the rotation and revolution are in the same direction, the critical value of eccentricity decreases.
\end{abstract}

\section{Introduction}

In the process of oil production in surface-driven screw pump wells, the movement of sucker rod in the tubing is rotation around its own axis and revolution around the tubing axis[1]. Previous studies on flow laws in eccentric annuli mainly focused on Couette flow induced by eccentric rotation of inner rod[2-4], Poiseuille flow in eccentric annuli[5-10], and spiral flow combined with both[11-16]. These studies have a large gap between the working conditions of the screw pump rod string and the drill string rotating in the drilling fluid, and the research on the complex flow field induced by the planetary motion of the inner rod in the circular tube that is close to the actual working condition very few[17]. Among them, Cui Haiqing [18-23]transformed the equation into a bipolar coordinate system for solution, and performed detailed calculations on the eccentric annular flow field of the inner rod executing a planetary motion. However, these studies are all solved under the condition of ignoring the inertial force. When the rotation speed is large, there may be large errors. Yang Shuren [24-26] used Ansys Fluent software to numerically simulate the spiral flow, and conducted a more complete simulation analysis of the flow field and the force on the inner rod, but did not simulate the eccentric annular Couette flow induced by the inner rod executing a planetary motion Li Yinpeng [28] proposed the idea of solving the fluid flow in the annulus of the inner rod executing a planetary motion based on the superposition principle, which simplified the plane flow field induced by the inner rod executing a planetary motion and solved it. The particle image velocimetry (PIV) method was used to test the flow field of the inner rod eccentric rotation and the outer cylinder rotation. In order to further improve the understanding of eccentric annular flow field, increase oil production and reduce energy consumption. The author numerically simulates the plane flow field induced by the eccentric rotation of the inner rod and the rotation of the outer cylinder, what's more, we simulate the plane flow field induced by the planetary motion of the inner rod. The fluid force on the inner rod when the inner rod moves in the outer cylinder is analyzed.

\footnotetext{
*Corresponding author: ysudshm@163.com

1936025628@qq.com
} 


\section{Simulation of flow field in eccentric annulus with inner rod and outer cylinder rotating}

\section{1 simulation model}

The planetary movement of the inner rod in the outer tube is shown in Figure 1(a). The eccentric annulus composed of the inner rod and the outer tube is filled with fluid, and the outer tube is stationary. The inner rod revolves around the axis of the outer tube at an angular velocity $\Omega$ and at the same time rotates around its own axis at an angular velocity $\omega$, driving the fluid to flow in the eccentric annulus. According to the literature [28], the planetary motion model of the inner rod is simplified to the equivalent mechanical model of eccentric rotation of the inner rod and the rotation of the outer cylinder as shown in Figure 1(b). The equivalent mechanical model is divided into two motions, the first movement is that the inner rod rotates around its own axis at a certain angular velocity $\Omega$ and revolves around the axis of the outer cylinder at the same angular velocity. At the same time, the outer cylinder also rotates at the same angular velocity; The second motion is that the inner rod rotates eccentrically at an angular velocity of $\omega-\Omega$ and the outer cylinder rotates at an angular velocity of $-\Omega$. The planetary movement of the inner rod in the outer cylinder is realized by the superposition of two motions, where $\mathrm{R}$ is the radius of the outer tube, $r$ is the radius of the inner rod, and e is the eccentricity.

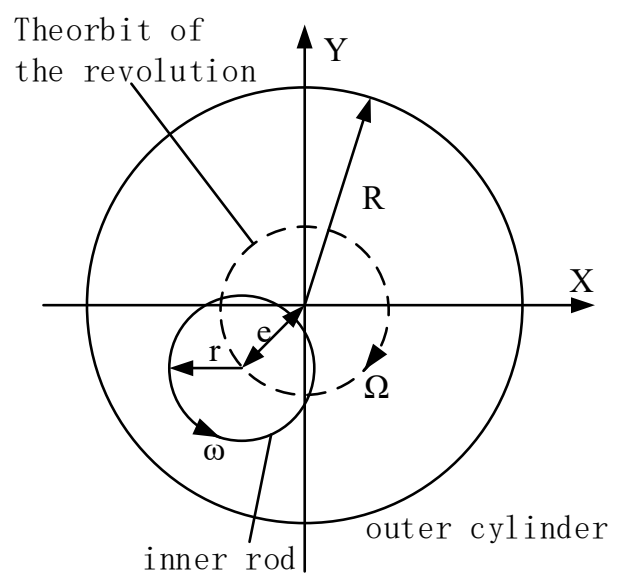

(a)
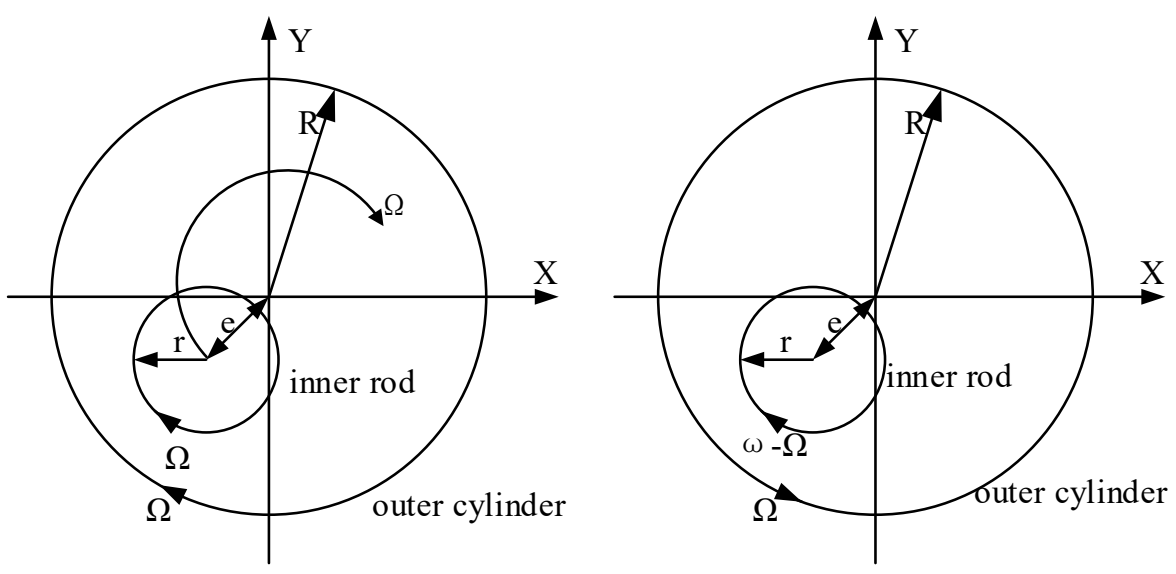

(b)

Fig.1. Schematic diagram of inner rod executing a eccentric motion

\subsection{Analysis of simulation results of annular flow field}

$\mathrm{R}=34.5 \mathrm{~mm}, \mathrm{r}=12.5 \mathrm{~mm}$, and the eccentricity is $0.2,0.4,0.6$, 0.8 . For the inner rod rotating in the same direction as the outer tube, the inner rod rotating speed is $60 \mathrm{r} / \mathrm{min}$ and the outer tube rotating is $120 \mathrm{r} / \mathrm{min}$; When the inner rod and the outer tube rotate in the opposite direction, speed of the inner rod's rotation is $60 \mathrm{r} / \mathrm{min}$ and the outer tube's rotation is $60 \mathrm{r} / \mathrm{min}$. Set density of fluid as $1150 \mathrm{~kg} / \mathrm{m} 3$ and viscosity as $0.012 \mathrm{pa} \cdot \mathrm{s}$. 


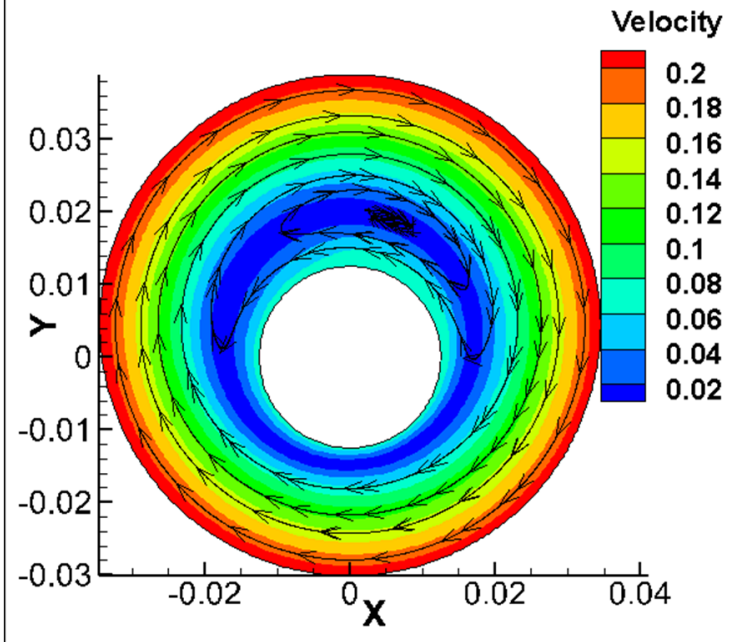

(a) $\varepsilon=0.2$

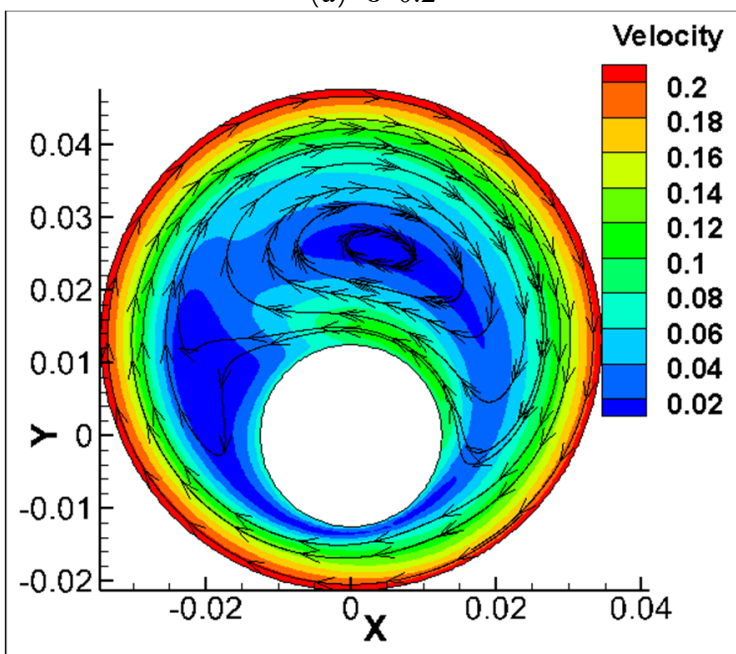

(c) $\varepsilon=0.6$

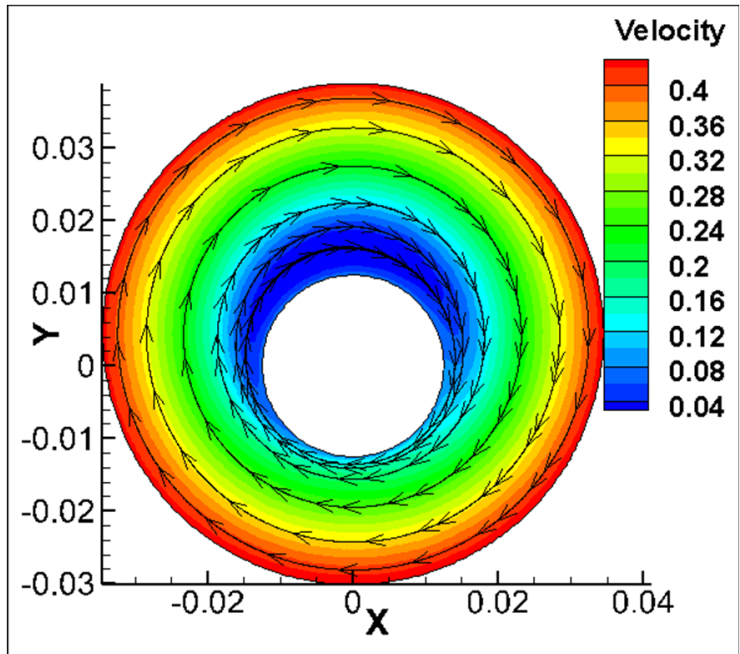

(e) $\varepsilon=0.2$

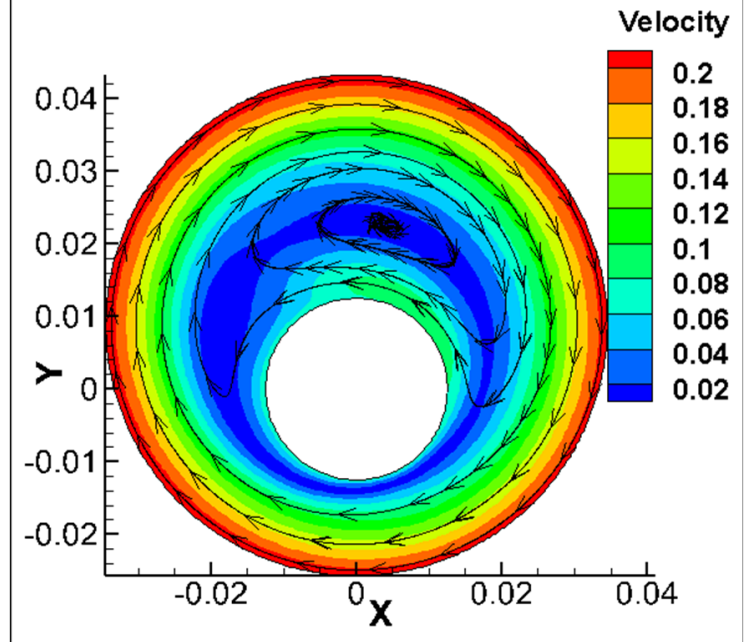

(b) $\varepsilon=0.4$

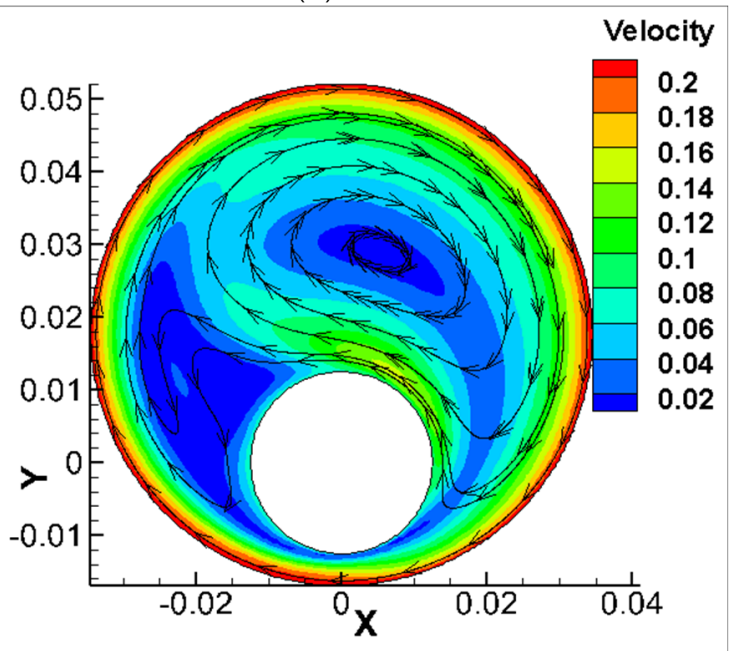

(d) $\varepsilon=0.8$

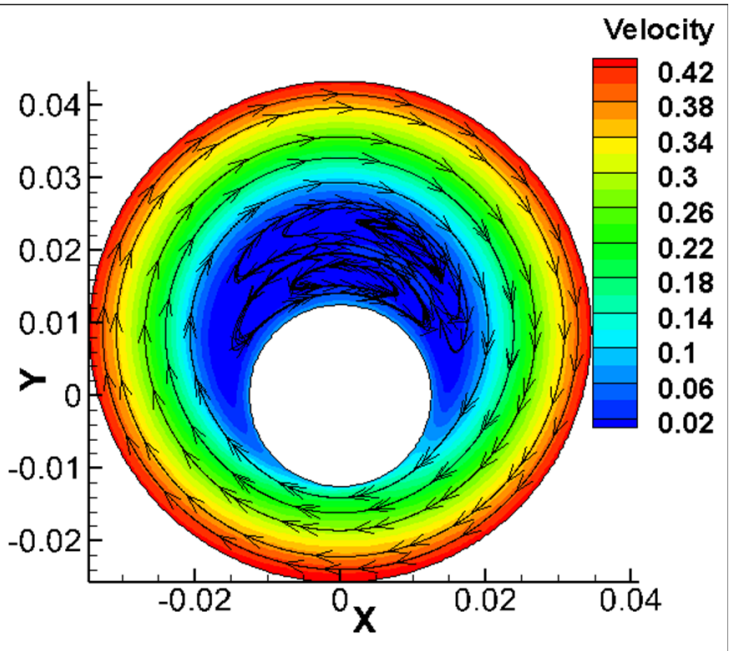

(f) $\varepsilon=0.4$ 


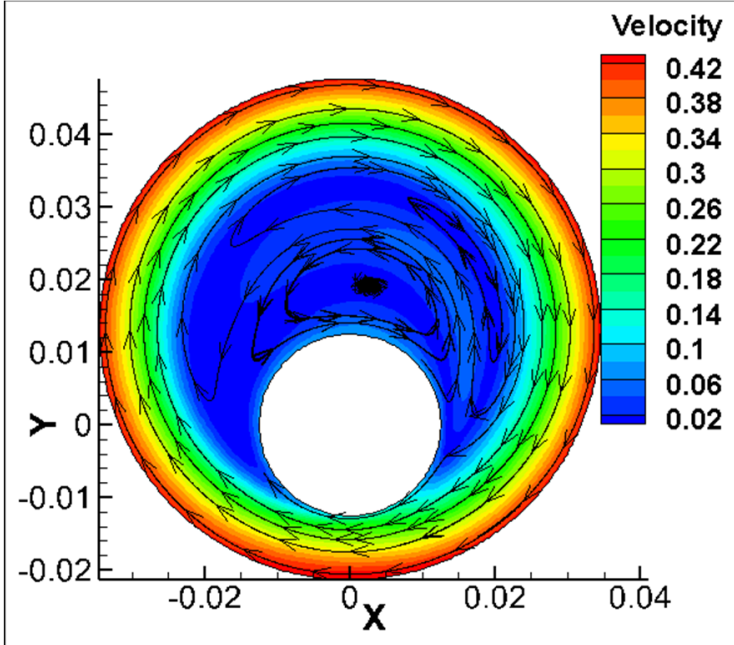

(g) $\varepsilon=0.6$

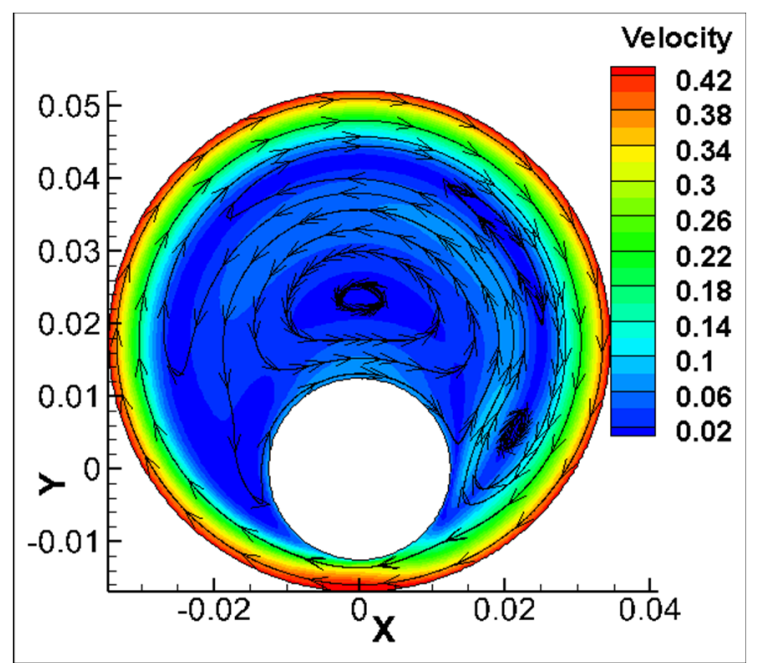

(h) $\varepsilon=0.8$

Fig.2. Velocity cloud map and streamline diagram under different eccentricity

By comparing the velocity cloud diagram and streamline diagram of the flow field with different eccentricities when the inner rod and the outer tube rotate in the same direction in Fig. 2(a d), it can be known that (1) as the eccentricity increases, the low-speed area of the fluid in the field is also increasing. Due to the viscous force of the fluid, the maximum value of the velocity appears near the wall of the outer tube and is close to the rotation speed of the outer tube; (2) When the eccentricity is small, the flow field does not appear the secondary flow, as the eccentricity increases, the phenomenon of secondary flow appears in the flow field, and there is more than one core of the secondary flow.

The analysis of the flow field velocity cloud diagram and streamline diagram under different eccentricities when the inner rod and the outer tube rotate in the opposite direction in Figure 2 (e $\sim$ ) shows that (1) When the inner rod and the outer tube rotate in the opposite direction, even if the eccentricity is very small, secondary flow will also occur. (2) With the increase of eccentricity, the secondary flow area increases more obviously, and there is always only one secondary flow core.

\subsection{Comparison between simulation results and experimental results}

The above simulation results of the model with eccentric rotation of inner rod and rotation of outer tube are compared with the experimental results based on particle image velocimetry (PIV) method in reference [28]. The flow field obtained by the two methods is same, which proves the correctness of the simulation model and the simulation results.

\section{Flow field simulation of inner rod executing a planetary motion}

\subsection{Dynamic grid and UDF technology}

In Fluent, the angular velocity of inner and outer boundaries can be directly set for the simple model of eccentric rotation of inner rod and rotation of outer tube. However, the dynamic grid model should be adopted for the complex model of inner rod planetary motion with violent boundary motion. The moving mesh mainly includes the description of the motion mode and the processing of the mesh, and the inner boundary's planetary motion is compiled by using UDF. Call the DEFINE_CG_MOTION macro to realize the rigid body motion of the boundary, and use vel[] and omega[] to specify the translation and rotation speed of the inner rod respectively, in which the revolution speed can be obtained by formula (1), and the planetary motion of the boundary can be realized through the combination of revolution and rotation. Formula (2) is the conservation equation of flux $\varphi$, which controls the body surface area $v$. Because the movement of boundary will definitely affect the original mesh, and if the mesh is not processed, it will produce negative volume. Therefore, the grid needs to be updated in the iterative calculation process. The update method chooses Spring and Remeshing. Control the mesh reconstruction by setting the Minimum Length Scale, Maximum Length Scale, and Maximum Cell Skewness. When the grid size is less than the minimum grid size or greater than the maximum grid size, the grid reconstruction is triggered.

$$
\begin{gathered}
\left\{\begin{array}{l}
V_{X}=-e \omega \sin (\omega t) \\
V y=e \omega \cos (\omega t)
\end{array}\right. \\
\frac{d}{d t} \int_{V} \rho \varphi d V+\int_{\partial V} \rho \varphi\left(u-u_{S}\right) d A= \\
\int_{\partial V} \Gamma V \varphi d A+\int_{V} S_{\varphi} d V
\end{gathered}
$$

In which $\omega$ is the rotational speed; $\rho$ is the density of the fluid; $u$ is the fluid velocity vector; $u s$ is the mesh deformation velocity of the moving mesh; $\Gamma$ is diffusion coefficient; $A$ is the control body surface area; $S \varphi$ is the source term of flux; $\partial V$ is the boundary of the control body. 


\subsection{Flow field analysis of inner rod planetary motion}

In the plane annular flow field induced by inner rod

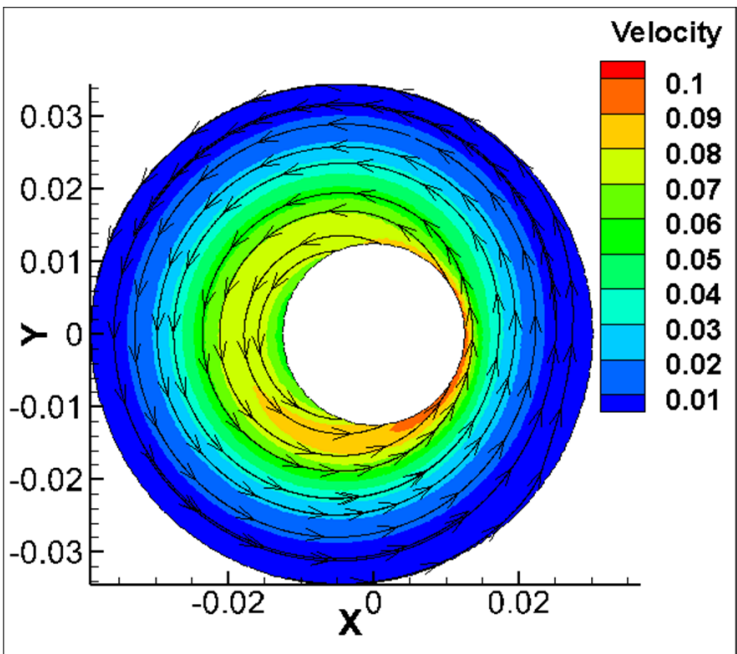

(a) $\varepsilon=0.2$

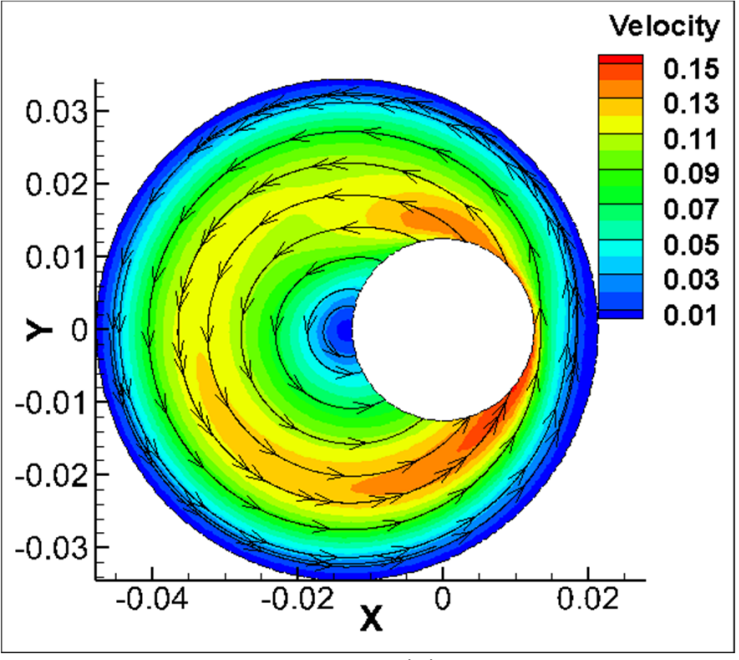

(c) $\varepsilon=0.6$

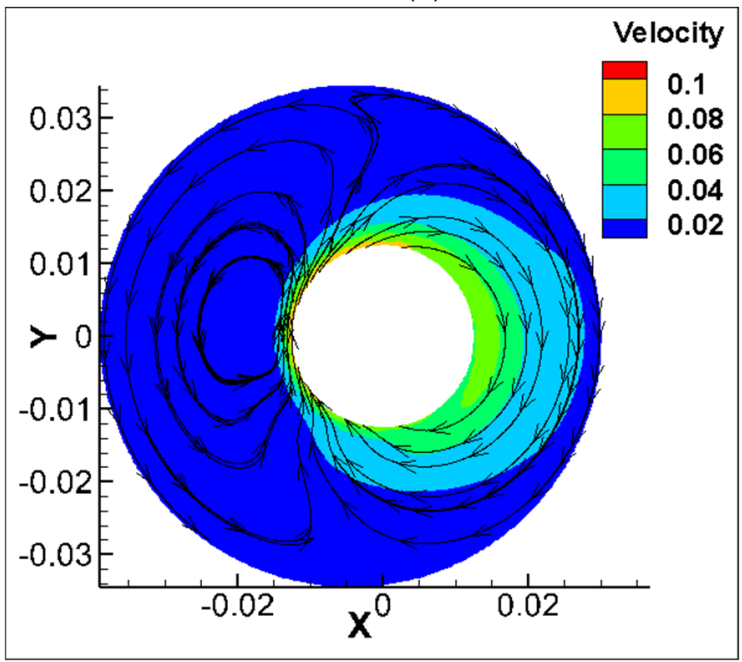

(e) $\varepsilon=0.2$ planetary motion, the rotational speed of inner rod rotation and revolution is $60 \mathrm{r} / \mathrm{min}$, and eccentricity $\varepsilon$ gradually increases at $0.2,0.4,0.6$ and 0.8 , respectively.

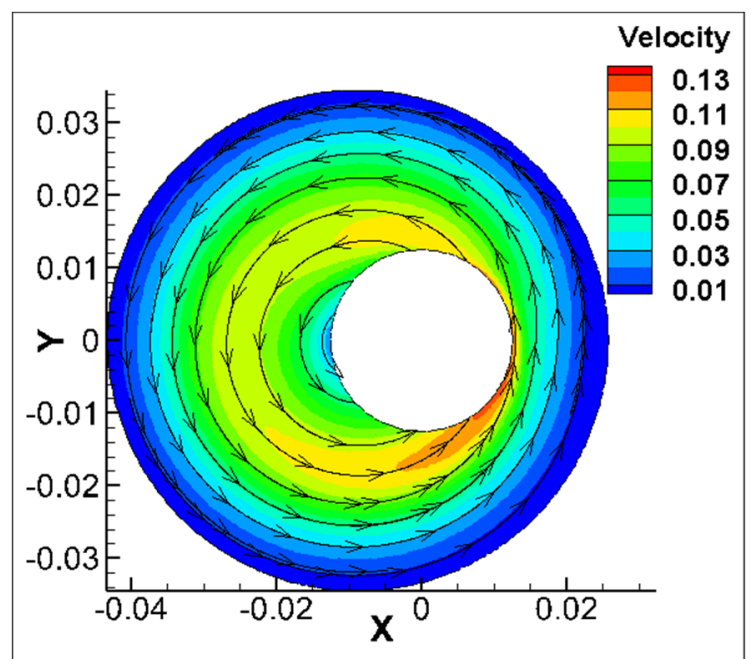

(b) $\varepsilon=0.4$

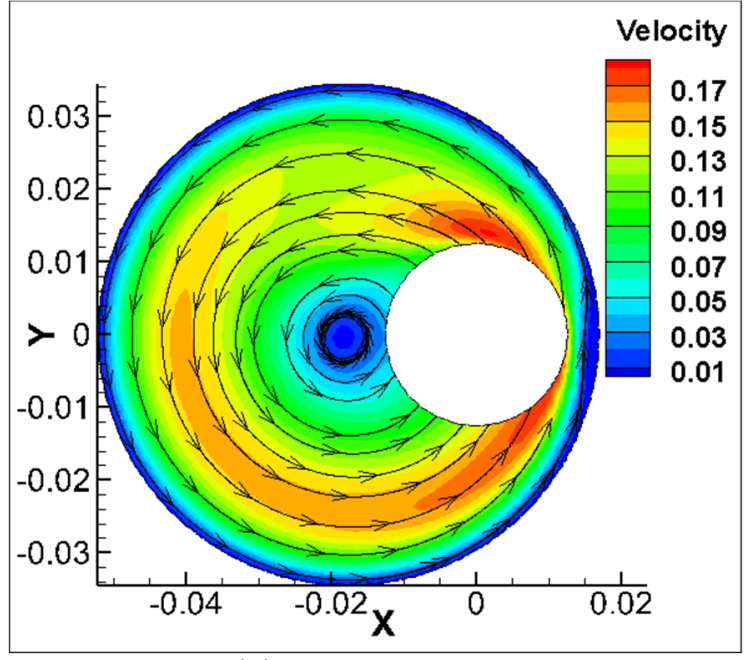

(d) $\varepsilon=0.8$

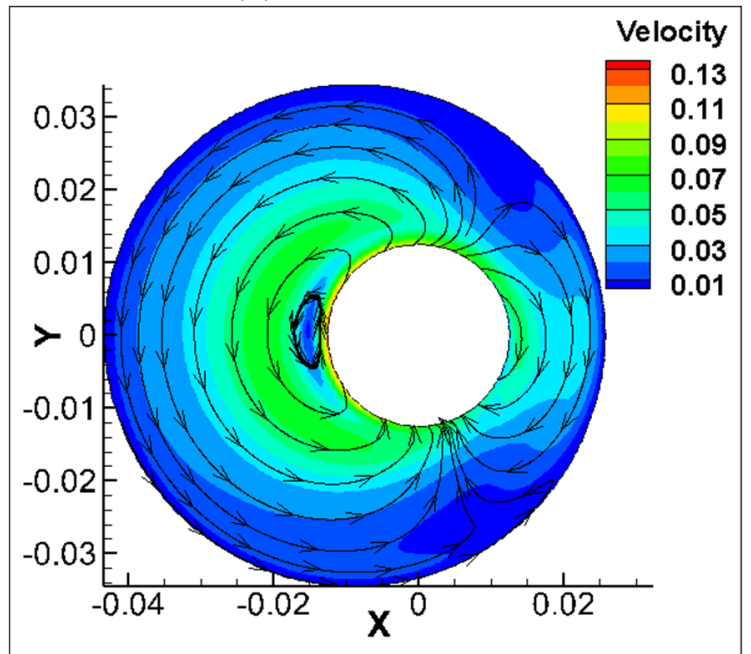

(f) $\varepsilon=0.4$ 


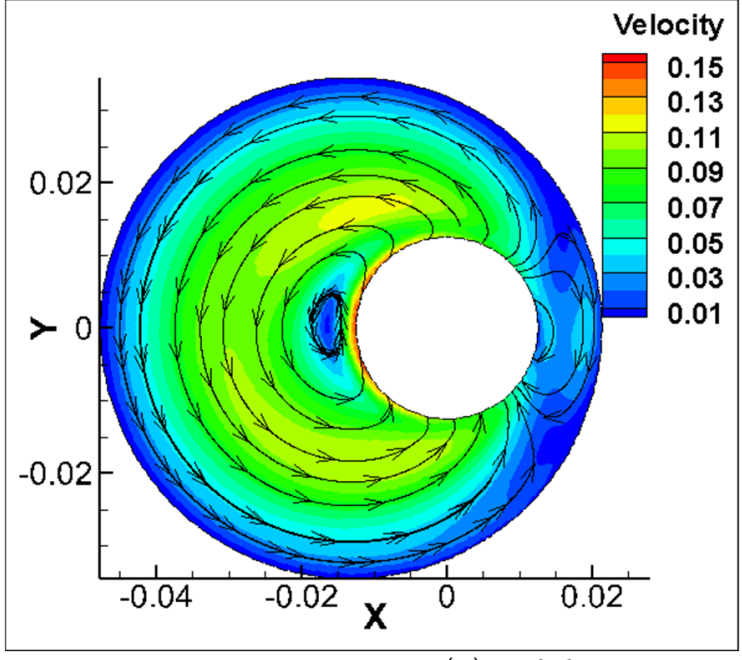

(g) $\varepsilon=0.6$

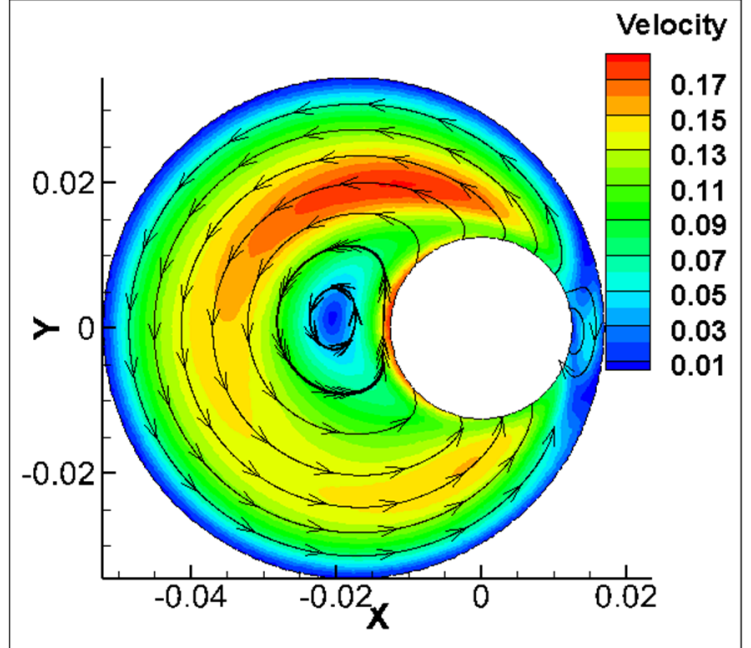

(h) $\varepsilon=0.8$

Fig.3. Velocity cloud map and streamline diagram under different eccentricity

\subsubsection{Flow field analysis.}

As shown in Figure 3(a d), the annulus plane flow field velocity cloud diagram and streamline diagram under different eccentricity conditions when the inner rod rotates and revolves in the same direction. The flow velocity near the wall of the outer tube in the flow field is close to zero, and the high-speed zone is distributed on the semicircular orbit outside the inner rod; With the increase of eccentricity, the low velocity region appears at the outer tube axis, and the obvious secondary flow phenomenon appears when the eccentricity is about 0.8 .

It can be seen from Figure 3(e h) that when the rotation direction of the inner rod is clockwise and the

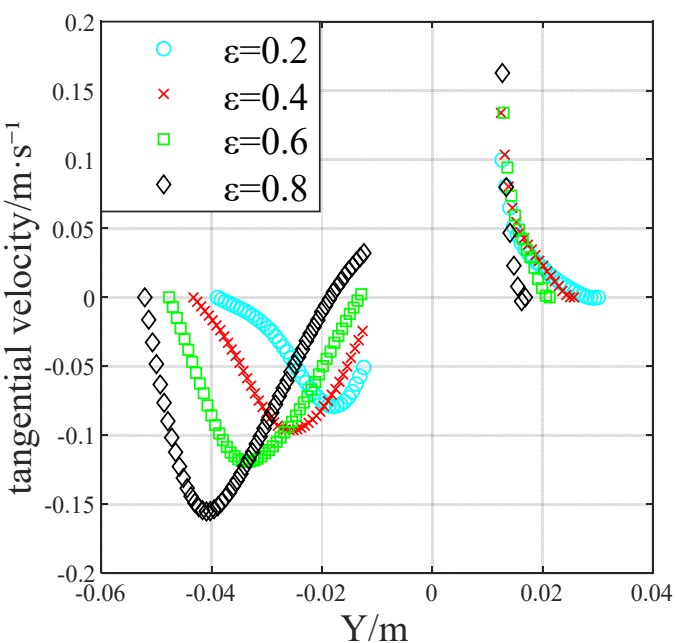

(a) same direction direction of revolution is counterclockwise, secondary flow appears in the wide gap of the annulus and near the wall of the inner rod, and the core of the secondary flow is always one; It can be known from the streamline distribution that the fluid flow direction in the wide gap area of the annulus is counterclockwise, and the fluid flow direction in the narrow gap area is clockwise.

On the line connecting the axis of the inner rod and the outer tube, 100 points in the eccentric annulus fluid area are selected, extracting the tangential velocity of the fluid at that position, and draw the relationship between the tangential velocity and the position as shown in Figure 4.

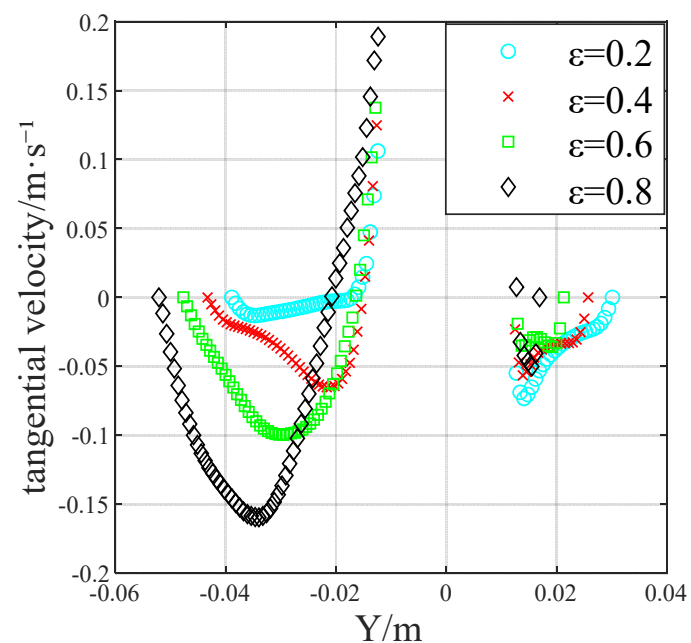

(b) reverse direction

Fig.4. The tangential velocity of fluid under different eccentricity

It can be seen from Figure 4(a) that when the inner rod's rotate in the same direction as the revolution, the tangential velocity of the fluid in the wide gap of eccentric annulus is negative, and the fluid in the narrow gap is positive; The fluid velocity distribution near the wall surface of the inner rod is that the velocity in the narrow space side is large and the velocity in the wide space side is small, which is due to the superposition of rotation velocity and revolution velocity in the narrow gap side of the inner rod, and the opposite direction of velocity in the wide gap side of the inner rod cancels each other.

For Figure 4 (b), when the rotation and revolution of the inner rod are reversed, the tangential velocity near the outer tube approaches zero; due to the superposition of the rotation velocity and revolution velocity, the maximum velocity of the fluid appears in the wide gap of the annulus 
and near the wall of the inner rod; in the narrow gap of the annulus near the wall of the inner rod, the rotation velocity and revolution velocity cancel each other , and the velocity of fluid is small.

\subsubsection{Force analysis.}

The pressure cloud diagram of the eccentric annulus flow field induced by the inner rod executing a planetary motion is shown in Figure 5. For the case of rotation in the same direction, according to the pressure cloud diagram shown in Figure $5(\mathrm{a} \sim \mathrm{d})$, it can be seen that the high pressure area is mainly distributed in the area near the outer tube with a wide gap, where the maximum pressure appears in the orbital forward flow on the narrow side of
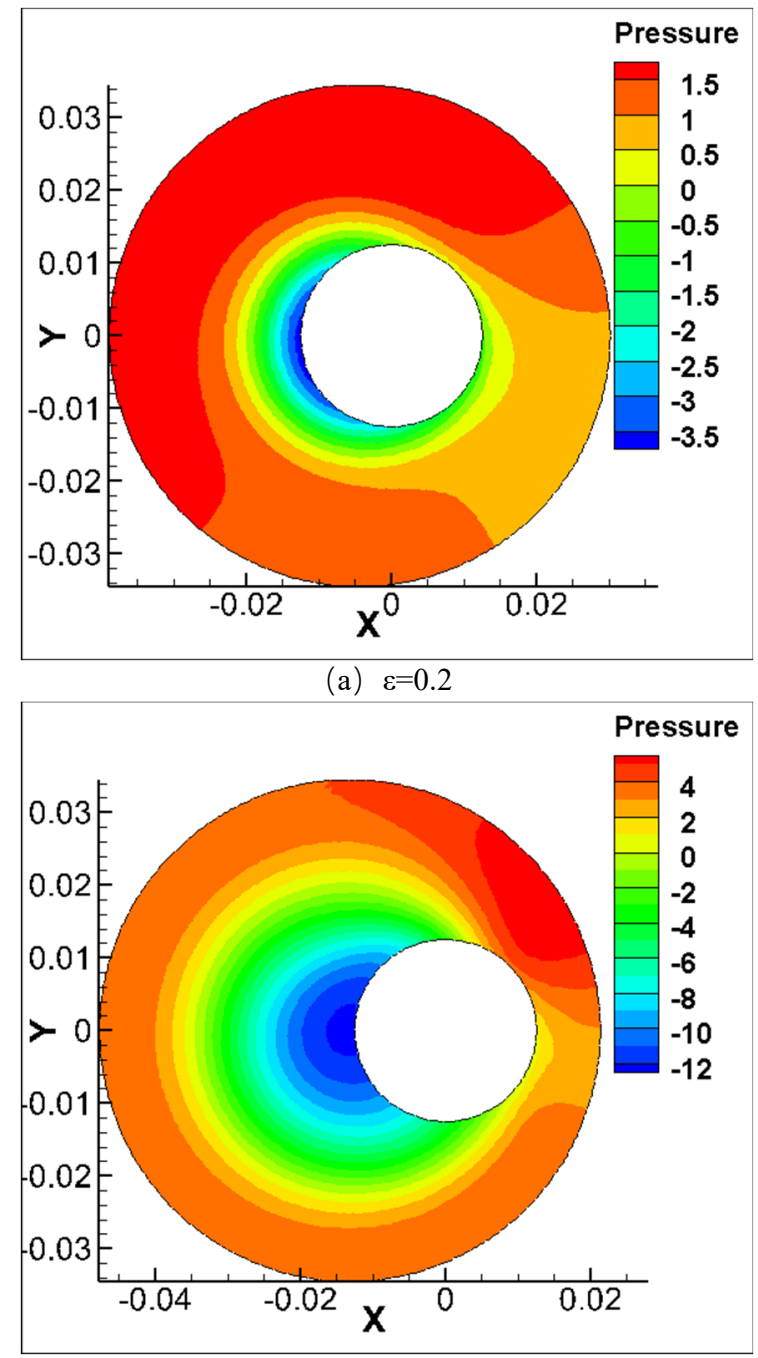

(c) $\varepsilon=0.6$ the surface, there is a tendency to prevent the inner rod from revolving and push the inner rod back toward the axis of the outer tube; The low pressure area appears at the wide gap of the annulus near the inner rod, and the low pressure area also increases with the increase of eccentricity.

According to the pressure cloud diagram of the reverse rotation of the inner rod shown in Figure $5(\mathrm{e} \sim \mathrm{h})$, it can be known that the high pressure area is concentrated in the wide gap area of the annulus when the eccentricity is small, and there is a tendency to push the inner rod outward; With the increase of eccentricity, a high pressure area appears at the narrow gap of the revolving front face of the inner rod, and a low pressure area appears at the axis of the outer tube. Due to the pressure difference, the inner rod has a tendency to push back and prevent its revolution.

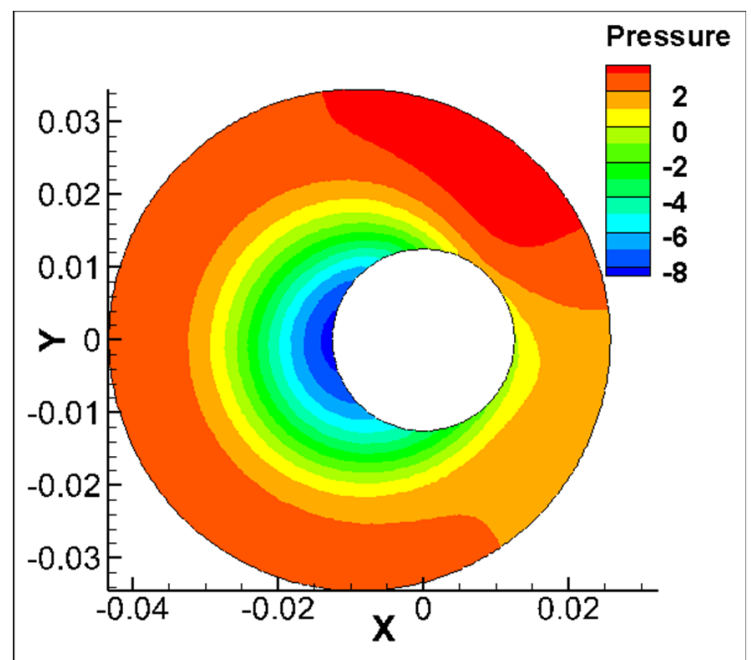

(b) $\varepsilon=0.4$

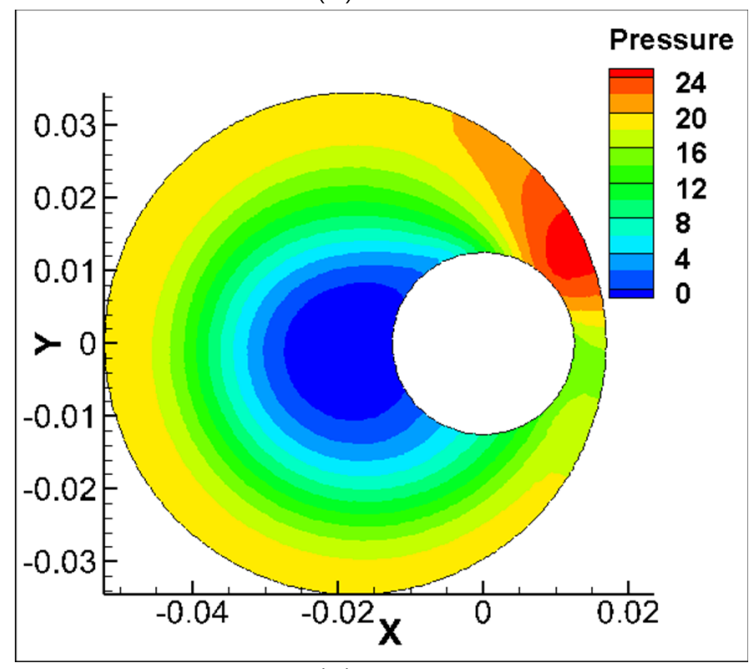

(d) $\varepsilon=0.8$ 


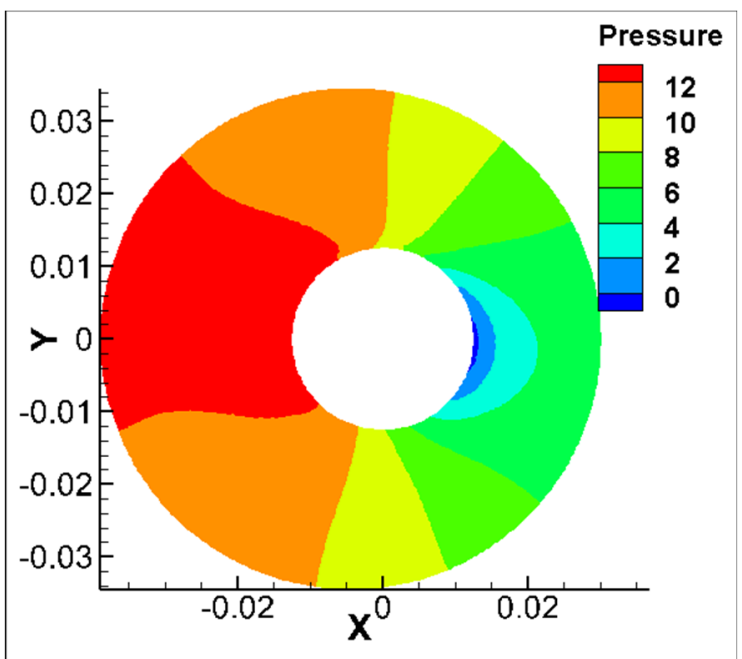

(e) $\varepsilon=0.2$

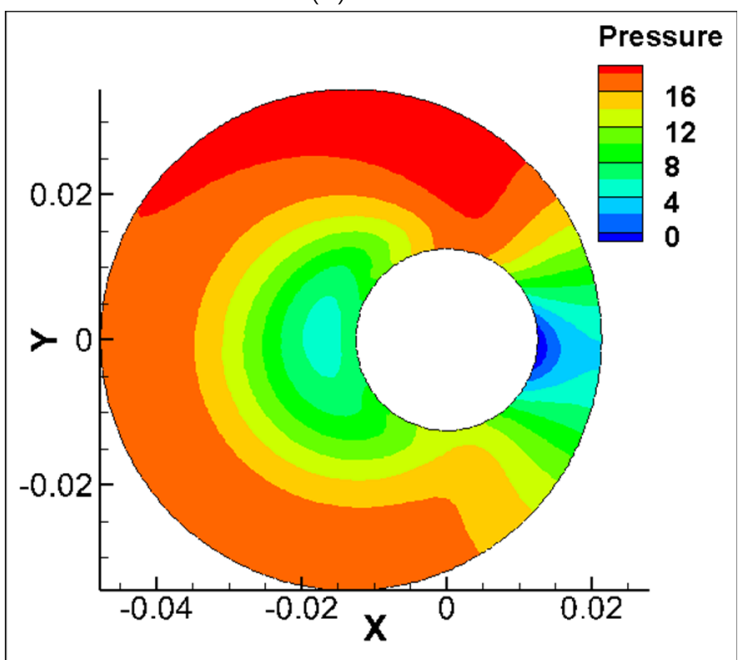

(g) $\varepsilon=0.6$

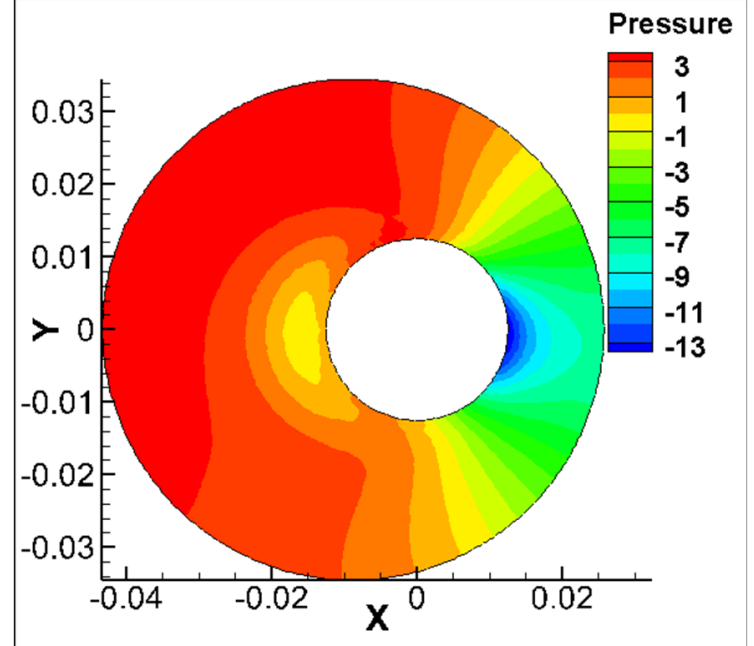

(f) $\varepsilon=0.4$

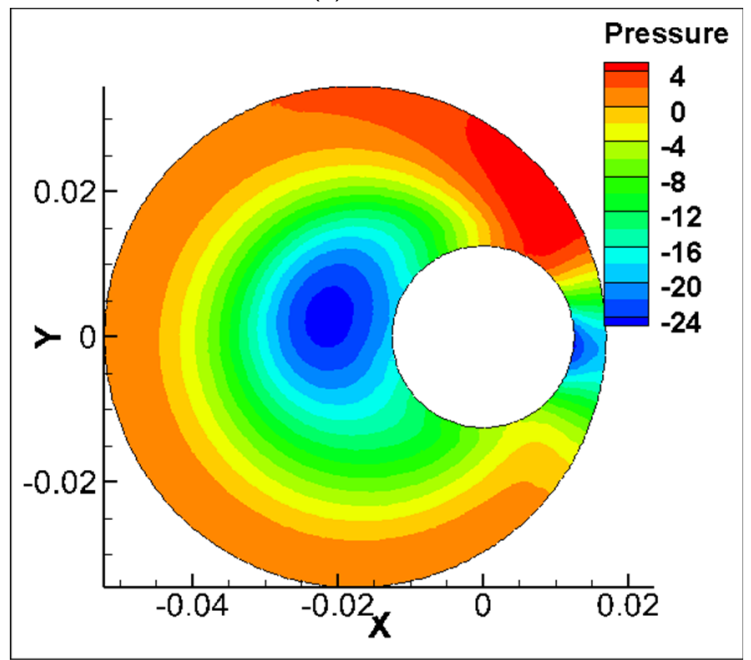

(h) $\varepsilon=0.8$

Fig.5. Pressure nephogram under different eccentricity

Figure 6 is a graph showing the relationship between the radial and tangential decomposition of the fluid force on the inner rod per unit length and the eccentricity. (1) It can be seen from Figure (a) that for only the revolution, the rotation and the revolution in the same direction, the rotation and the revolution in the reverse direction have little effect on the tangential force of the inner rod, and the tangential force received increases with the eccentricity (2) For the change in the radial force on the inner rod shown in Figure (b), it can be seen that there is a critical value of eccentricity (about 0.4 ), when the eccentricity is less than this critical value, the rod is pushed outward, the rod is pushed inward when it is greater than the critical value; (3) When the rotation is reversed, the critical value of eccentricity increases (approximately 0.72); (4) When the rotation and revolution are in the same direction, the critical value of eccentricity decreases, so that the radial force on the inner rod is always negative, that is, the inner rod has a tendency to be pushed inward at different eccentricities. 


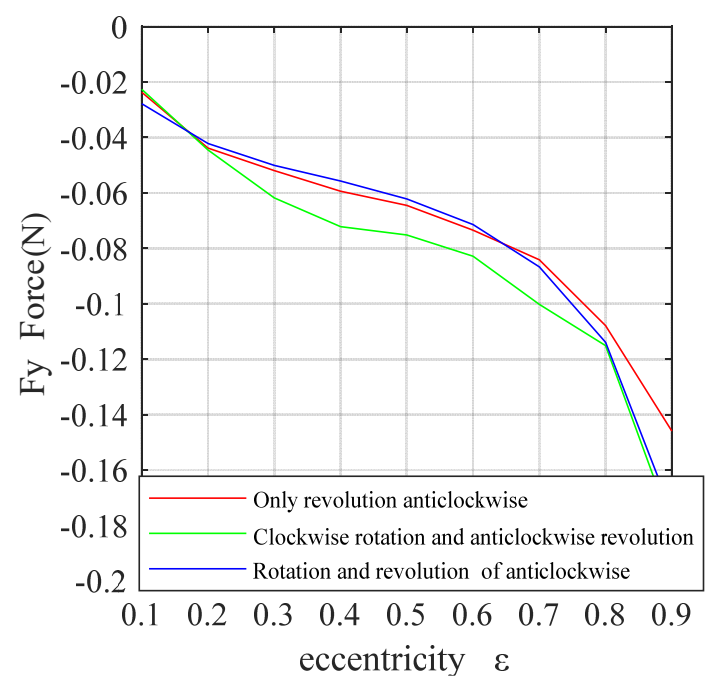

(a) Tangential force

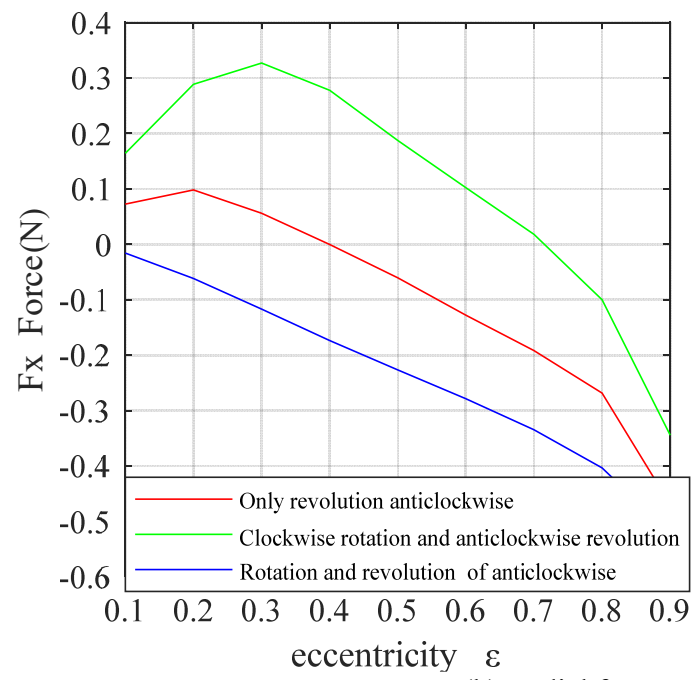

(b) Radial force

Fig.6. Relationship between fluid force on the inner rod and eccentricity

\subsection{Flow field simulation of inner rod executing a planetary motion based on superposition principle}

According to the superposition principle, the first motion is eccentric rotation with the angular velocity of inner rod is $60 \mathrm{R} / \mathrm{min}$, and the second motion is rotation with the angular velocity of outer cylinder is $-60 \mathrm{R} / \mathrm{min}$.

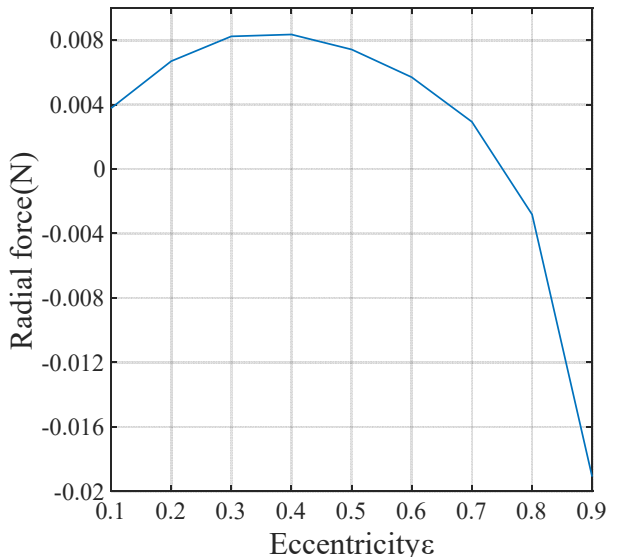

\subsubsection{Force analysis}

According to the relationship between force and eccentricity when the inner rod executing a planetary movement based on the superposition principle shown in Fig. 7, it can be seen that the tangential force of the inner rod increases with the increase of eccentricity, There is a critical value of eccentricity, which changes the direction of the radial force on the inner rod.

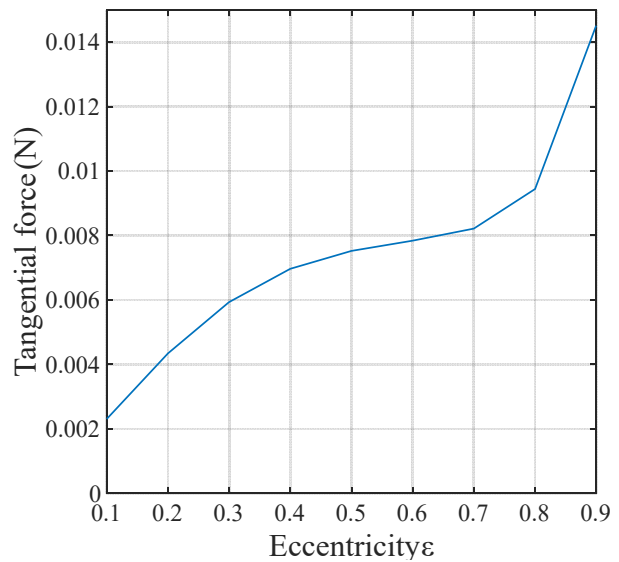

(a) Only the inner rod executing a rotation eccentrically 

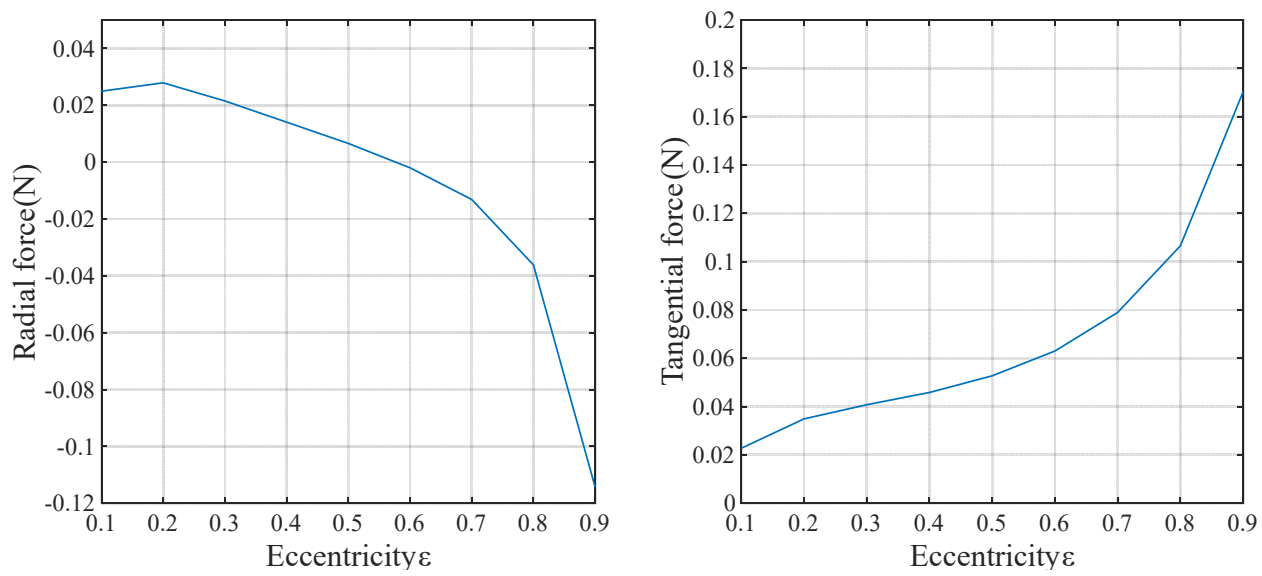

(b) Only the outer cylinder rotates

Fig.7. Relationship between fluid force on the inner rod and eccentricity

\section{Conclusion}

(1) Through the comparative analysis of simulation and experimental results, it can be known that it is feasible to study the eccentric annulus flow field under complicated working conditions through numerical simulation.

(2) When the rotation of the inner rod is in the same direction as the revolution, the fluid velocity near the wall surface of the inner rod is greater on the narrow-spacing side of the annulus and smaller on the wide-spacing side. When the eccentricity is greater than the critical value, a secondary flow occurs; when the inner rod rotates in the opposite direction to the revolution, the fluid velocity near the wall of the inner rod is greater on the wide-spacing side of the annulus and slower on the narrow-spacing side. Different eccentricities have obvious secondary flow phenomenon, and the secondary flow is now in a wide gap and close to the inner rod.

(3) When the inner rod revolves only, the eccentricity of the inner rod is pushed outward by the force of the fluid when it is less than the critical value, and the inner rod is pushed inward when the eccentricity of the inner rod is less than the critical value; when the rotation and revolution are reversed, the critical value of eccentricity increases; when the rotation and revolution are in the same direction, the critical value of eccentricity decreases.

\section{Acknowledgements}

The work is sponsored by the National Natural Science Foundation of China (No. 51974276)

\section{References}

1. Ji Haijun. Flow of a Power law fluid in annulus with the inner cylinder executing a planetaey motion[D]. Daqing: Daqing Petroleum Institute, 2005.

2. B. Y. Ballal,R. S. Rivlin. Flow of a viscoelastic fluid between eccentric cylinders[J]. Rheologica Acta, 1979, 18(3).
3. C. Shu,L. Wang,Y.T. Chew,N. Zhao. Numerical study of eccentric Couette-Taylor flows and effect of eccentricity on flow patterns[J]. Theoretical and Computational Fluid Dynamics,2004,18(1).

4. Dana Grecov,Jean-Robert Clermont. Numerical simulations of non-Newtonian flows between eccentric cylinders by domain decomposition and stream-tube method[J]. Journal of Non-Newtonian Fluid Mechanics,2004,126(2).

5. Zhao Yinghai. New method for studying fluid flow in eccentric annulus[J]. Chinese Journal of Applied Mechanics, 1997(03): 107-111+154.

6. $\mathrm{Xu}$ Jianping, Chen Qinlei, Huo Zilun. Velocity distribution of non-Newtonian fluid through eccentric annulus[J]. Well Testing, 2000(03): 20-21+40-91.

7. Yang Yuanjian. Unsteady flow of Viscoelastic fluid in eccentric annuli with inner cylinder reciprocating axially[D]. Daqing: Daqing Petroleum Institute, 2005.

8. Chen Wan. Numerical simulation of flow field in eccentric annulus[D]. Daqing: Daqing Petroleum Institute, 2009.

9. Fan Fan. Generalized hydraulic calculation method for non-Newtonian fluid in eccentric annulus[J]. Inner Mongolia Petrochemical Industry, 2018, 44(03): 49.

10. Din Yuxi. Numerical simulation of unsteady flow of power-law fluid in the eccentric annulus[D]. Daqing: Northeast Petrleum University, 2018.

11. M. Zidan,A. Abu-El Hassan. Flow of a viscoelastic fluid between eccentric cylinders[J]. Rheologica Acta, 1985,24(2).

12. Zheng Yingren. Annular spiral flow field of Bingham liquid[J]. Journal of Oil and Gas Technology, 1988(02):38-45+6.

13. Zhang Haiqiao, Wu Jizhou. Analytical solution of non-Newtonian fluid eccentric annulus spiral flow [J]. Applied Mathematics andMechanics, 1994(07): 627638. 
14. Xing Jun, Han Xueson, Han Yankai, et al. PIV test research of eccentric annulus spiral flow [J]. Offshore Oil, 2009,29(03): 107-112.

15. Wang Xiaobing, LiuYang, Cui Haiqing, et al. Experimental Research on the spiral flow restraining eccentric wear of the sucker and tubing with PIV [J]. Engineering Mechanics, 2011, 28(11):225-30.

16. Zhao Yan. Non-newtonian fluid in eccentric annulus helical flow field of the CFD simulation[D]. Hang Zhou: China Jiliang University, 2017.

17. J. Y. Kazakia,R. S. Rivlin. Flow of a viscoelastic fluid between eccentric rotating cylinders and related problems[J]. Rheologica Acta,1977,16(3).

18. Cai Meng, JiHaiqing, Pei Xiaohan, Li Ziwen, et al. The velocity distribution of law fluid flowing in annulus with inner cylinder executing a planetary motion[J]. Journal of Daqing Petroleum Institute, 2008(03): 12-17+133-134.

19. Zhang Bin, Cui Haiqing, Cui Haiqing, et al. Numerical calculation of flow rate of law fluid flowing in annulus with inner cylinder executing a planetary motion[J]. Journal of Harbin Engineering University, 2010,31(08):1105-1108.

20. Cui Haiqing, Pei Xiaohan, Cai Meng. The secondary flow in power law fluid flow in annulus with the inner cylinder executing a planetary motion $[\mathrm{J}]$. Acta Petrolei Sinca, 2007(01): 134-138.

21. Cui Haiqing, Xiu Deyan, Pei Xiaohan, et al. The normal stress distribution on the inner cylinder force by power law fluid flowing in annulus with the inner cylinder executing a planetary motion $[\mathrm{J}]$. Journal of China University of Petroleum(Edition of Natural Science), 2007(01): 67-71.

22. Cai Meng, Cui Haiqing, Pei Xiaohan, et al. Analysis of the force on the inner cylinder wall when the power law fluid flowing in annulus with the inner cylinder executing a planetary motion[J]. Journal of Daqing Petroleum Institute,2008(05): 9-22+122-123.

23. Cui Haiqing, Zhang Shuyun, Xiu Deyan, et al. The pressure gradient in Newtonian fluid flow in annulus with the inner cylinder executing a planetary motion[J]. Journal of China University of Petroleum(Edition of Natural Science), 2008,32(06): 76-78+87.

24. Pang Boxue, Liao Xiaokui. Research on the influence of inner cylinder rotation and revolution on the flow of non-Newtonian fluid in eccentric annulus[J]. Contemporary Chemical Industry, 2015,44(05): 1082-1084+1089.

25. Pang Boxue, Yang Shuren, Liu Lili, et al. Numerical simulation of flow field in eccentric annulus whose inner tube has planetary mtion based on dynamic mesh and UDF[J]. New Technology \& New Process, 2015(6): 64-67.

26. Yang Shuren, Chen Can, Pang Boxue. Numerical simulation for the Power-Law fluid flows in annulus with planetary motion for the inner tube[J]. Natural Gas and Oil, 2017. 35(1): 89-93+11.
27. Li Zifeng, Li Jingyuan, Ma Xingrui, et al. The mathematical model of Newtonian liquid laminar flow induced by the rotation and revolution of the drill string[J]. Mechanics and Engineering, 1995,17(05): 51-53.

28. Li Yinpeng, Li Zifeng, Wang Changjin, et al. PIV analysis of plane flow field induced by the planetary motion of drill string based on superposition principle[J]. Acta Petrolei Sinica, 2018, 39(12): 1421$1428+143$ 